\title{
Compound heterozygous mutations in UBA5 causing early-onset epileptic encephalopathy in two sisters
}

\author{
Gudny A. Arnadottir', Brynjar O. Jensson', Sigurdur E. Marelsson², Gerald Sulem', Asmundur Oddsson',
} Ragnar P. Kristjansson", Stefania Benonisdottir ${ }^{1}$, Sigurjon A. Gudjonsson', Gisli Masson', Gudmundur A. Thorisson?', Jona Saemundsdottir ${ }^{1}$, Olafur Th. Magnusson ${ }^{1}$, Adalbjorg Jonasdottir ${ }^{1}$, Aslaug Jonasdottir ${ }^{1}$, Asgeir Sigurdsson?', Daniel F. Gudbjartsson ${ }^{1,3}$, Unnur Thorsteinsdottir ${ }^{1,4}$, Reynir Arngrimsson ${ }^{5}$, Patrick Sulem ${ }^{1 *}$ (D) and Kari Stefansson ${ }^{1,4}$

\begin{abstract}
Background: Epileptic encephalopathies are a group of childhood epilepsies that display high phenotypic and genetic heterogeneity. The recent, extensive use of next-generation sequencing has identified a large number of genes in epileptic encephalopathies, including UBA5 in which biallelic mutations were first described as pathogenic in 2016 (Colin E et al., Am J Hum Genet 99(3):695-703, 2016. Muona M et al., Am J Hum Genet 99(3):683-694, 2016). UBA5 encodes an activating enzyme for a post-translational modification mechanism known as ufmylation, and is the first gene from the ufmylation pathway that is linked to disease.

Case presentation: We sequenced the genomes of two sisters with early-onset epileptic encephalopathy along with their unaffected parents in an attempt to find a genetic cause for their condition. The sisters, born in 2004 and 2006, presented with infantile spasms at six months of age, which later progressed to recurrent, treatment-resistant seizures. We detected a compound heterozygous genotype in UBA5 in the sisters, a genotype not seen elsewhere in an Icelandic reference set of 30,067 individuals nor in public databases. One of the mutations, c.684G > A, is a paternally inherited exonic splicing mutation, occuring at the last nucleotide of exon 7 of UBA5. The mutation is predicted to disrupt the splice site, resulting in loss-of-function of one allele of UBA5. The second mutation is a maternally inherited missense mutation, p.Ala371Thr, previously reported as pathogenic when in compound heterozygosity with a loss-of-function mutation in UBA5 and is believed to produce a hypomorphic allele. Supportive of this, we have identified three adult Icelanders homozygous for the p.Ala371Thr mutation who show no signs of neurological disease.

Conclusions: We describe compound heterozygous mutations in the UBA5 gene in two sisters with early-onset epileptic encephalopathy. To our knowledge, this is the first description of mutations in UBA5 since the initial discovery that pathogenic biallelic variants in the gene cause early-onset epileptic encephalopathy. We further provide confirmatory evidence that p.Ala371Thr is a hypomorphic mutation, by presenting three adult homozygotes who show no signs of neurological disease.
\end{abstract}

Keywords: Epileptic encephalopathy, UBA5, Hypomorphic mutation, Exonic splicing mutation, Ufmylation, Case report

* Correspondence: patrick.sulem@decode.is

1'deCODE Genetics/Amgen, Inc., Sturlugata 8, 101 Reykjavik, Iceland

Full list of author information is available at the end of the article 


\section{Background}

Epileptic encephalopathies are a group of childhood epilepsies characterized by severe seizures or other paroxysmal electrical activities that contribute to impaired cerebral function [1]. Several causes of epileptic encephalopathies have been described, including cerebral structural abnormalities, inborn errors of metabolism, chromosomal anomalies, and single-gene mutations [1]. A large number of single-gene mutations causing epileptic encephalopathies have been identified through the recent, extensive use of next-generation sequencing [1]. Currently, over 65 genes are known in the early-onset epileptic encephalopathies, of which 20 were discovered in the last three years alone $[1,2]$.

Here, we describe compound heterozygous mutations in the recently identified epileptic encephalopathy gene, $U B A 5$, in two sisters with early-onset epileptic encephalopathy. To our knowledge, this is the first case of biallelic mutations in $U B A 5$ to be described since the initial reports of pathogenic biallelic variants in $U B A 5$ causing early-onset epileptic encephalopathy [3, 4]. In addition, we present three adult Icelanders homozygous for one of the mutations detected in the sisters, p.Ala371Thr. The three homozygous individuals show no signs of neurological disease, which confirms that p.Ala371Thr is acting as a hypomorphic mutation.

\section{Case presentation}

The two affected sisters were born in 2004 (II-2) and 2006 (II-3) (see pedigree, Fig. 1). Their father (I-1; American, white non-Hispanic, born in 1975), mother (I-2; Icelandic, born in 1977) and two brothers (II-1; born in 2002 and II4 ; born in 2014) are unaffected. The pregnancies were uneventful, both sisters were delivered full term with normal birth weight. They first became symptomatic at three months of age, showing failure to thrive and a decline in motor skills such as tracking and head support. The older sister (II-2) was admitted to the Children's Hospital of Iceland at six months of age because of increasing irritability, nutritional difficulties and episodes of infantile spasms. An electroencephalogram, performed at the time, showed modified hypsarrhythmia. Her seizures initially responded to vigabatrin, the infantile spasms disappeared and she became less irritable. After a few weeks however, the infantile spasms returned and she responded only partly to high doses of adrenocorticotropin. She developed severe intellectual disability with seizures and dystonic cerebral palsy and was diagnosed with early-onset epileptic encephalopathy.

The younger sister (II-3) had a similar clinical course, although slightly milder. She has severe intellectual disability, epilepsy, and quadriplegic cerebral palsy with extrapyramidal features, and received the same diagnosis as her sister of early-onset epileptic encephalopathy. A clinical examination of the two sisters, at ages 10 and 8 , showed mild dysmorphic

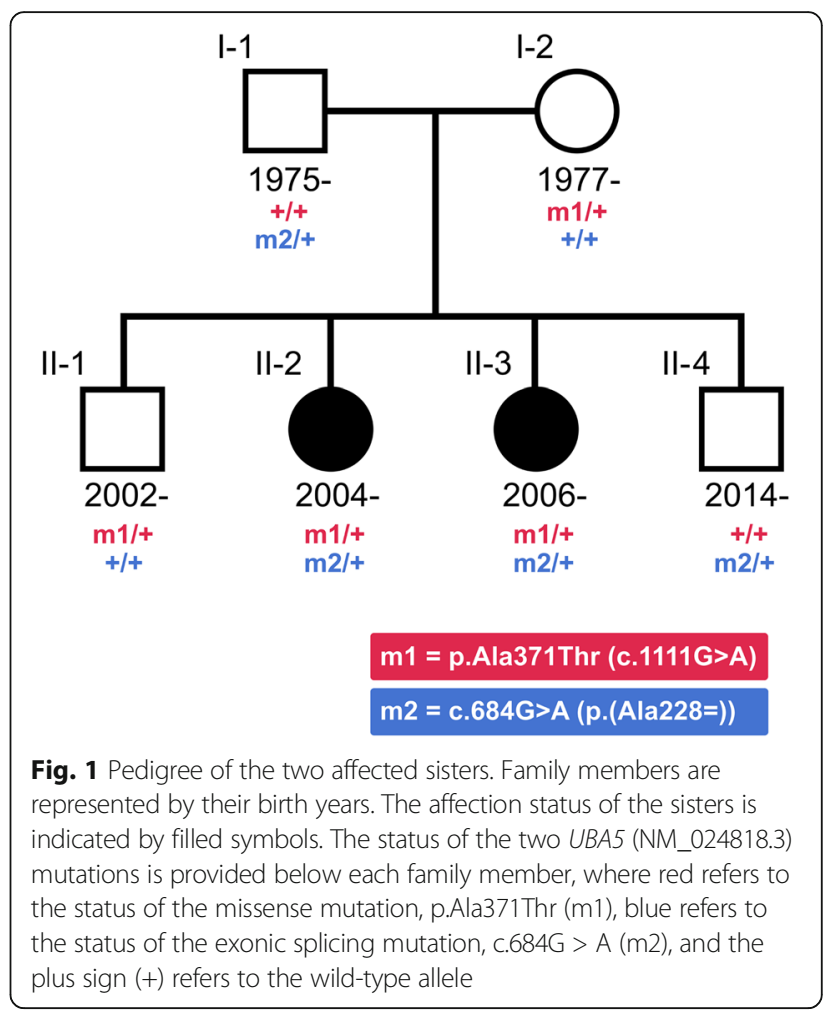

facial features, including a slightly upturned nose and mild hypertelorism, difficulties with visual fixation and following, and low muscle tone (although normal muscle strength proximally and distally). Opthalmologic examinations showed no abnormalities of the retina or optic nerves and normal reactions to strong light, bilaterally. An MRI, performed at ages 12 and 10, showed relatively wide sulci and mild cerebral atrophy (Additional file 1: Figure S1). At their latest examination, both sisters still had frequent seizures. They have shown limited response to several antiepileptic drugs in high doses (including high dose phenytoin, levetiracetam, and high dose vigabatrin). The CARE guidelines were followed in this case description.

In an attempt to find a genetic cause for their condition, we sequenced the genomes of the two sisters and their parents to a targeted depth of $30 \times$ (for a detailed description of the whole-genome sequencing see Additional file 2: Supplementary methods). The genetic analysis focused on rare genotypes at coding and splicing regions in the genome (SNPs and small indels). Large-scale sequencing of the Icelandic population by deCODE genetics $[5,6]$ has yielded a local normative set, currently consisting of 30,067 whole-genome sequenced Icelanders (to a median depth of over $30 \times$ ). The minor allele frequency of sequence variants was determined from this set of whole-genome sequenced Icelanders as well as from an international dataset of 138,632 individuals either whole-exome or whole-genome sequenced (the genome Aggregation Database, gnomAD [7]). We defined rare autosomal recessive genotypes as homozygous or compound 
heterozygous sequence variants, each with a minor allele frequency lower than $2 \%$ in our set and in gnomAD. We defined rare autosomal dominant genotypes as variants with a minor allele frequency lower than $0.1 \%$ in our set and gno$\mathrm{mAD}$. We assessed all possible modes of inheritance but considered a recessive mode of inheritance or a dominant mode of inheritance with parental germline/somatic mosaicism most likely, based on the manifestation of two affected siblings. Due to the extent of clinical similarity and the severity of their condition, we focused on genotypes shared by the two sisters.

We identified two rare genotypes that fulfilled the above criteria (Additional file 3: Figure S2 shows our approach to variant filtering). One of the genotypes was in MTA1, a gene not previously known to contribute to Mendelian disease (Additional file 3: Figure S2). The other was a compound heterozygous genotype in $U B A 5$, a gene that has only very recently been linked to an epilepsy phenotype [3, 4, 8] (Early Infantile Epileptic Encephalopathy 44, EIEE44, MIM: 617,132, Creation date: 09/27/2016). UBA5 encodes ubiquitin-like modifier activating enzyme 5 , a necessary component of a post-translational modification mechanism known as ufmylation [4]. Compound heterozygous mutations were first reported in $U B A 5$ in two Chinese siblings presenting with autosomal recessive cerebellar ataxia in February 2016 [8]. In September 2016, two reports describing the pathogenic role of $U B A 5$ biallelic variants in early-onset epileptic encephalopathy were published $[3,4]$.
We would like to point to high similarity between the two affected sisters in Iceland and the cases presented in these two reports $[3,4]$ (Table 1$)$.

The compound heterozygous genotype we detected consists of a paternal exonic splicing mutation, c.684G > A, and a maternal missense mutation, p.Ala371Thr, in $U B A 5$ (Table 2 and Fig. 1). Sanger sequencing confirmed the genotypes of the sisters and their parents. The two unaffected brothers (II-1 and II-4, Fig. 1 and Additional file 4: Table S1) were Sanger genotyped and each found to be heterozygous for only one of the mutations.

The paternally inherited mutation, c.684G > A, is a splice region mutation occurring at the last nucleotide of exon 7 of $U B A 5$ in the exonic part of the splice donor $\left(5^{\prime}\right)$ region (Additional file 5: Figure S3). It appears to be a very rare and recent mutation, absent from 30,067 whole-genome sequenced Icelanders and detected only once in gnomAD's set of 138,632 whole-exome/genome sequenced individuals [7]. The mutation affects a highly conserved nucleotide, both in terms of evolutionary conservation (Table 1) and with regards to the consensus sequence for human splice donor regions. Based on both the high conservation of this position and a predicted altered splicing motif (in silico predictions) we suggest that the mutation disrupts the donor splice site of exon 7 of $U B A 5$. Two possible scenarios can result from this disruption; A) skipping of the subsequent exon in UBA5 (exon 8) or multiple exons (exons 8-12) from the mature mRNA or $\mathrm{B}$ ) a read-through into intron 7

Table 1 Clinical features of the two affected sisters compared to reported phenotypes in cases of UBA5 early-onset epileptic encephalopathy

\begin{tabular}{|c|c|c|}
\hline & Affected sisters & UBA5 early-onset epileptic encephalopathy cases $[2,3]$ \\
\hline Pregnancy and delivery & Normal & Normal \\
\hline Failure to thrive & Yes & Yes \\
\hline $\begin{array}{l}\text { Central } \\
\text { Nervous } \\
\text { System }\end{array}$ & $\begin{array}{l}\text { Early-onset epileptic encephalopathy } \\
\text { Dystonic cerebral palsy } \\
\text { Hypotonia } \\
\text { Infantile spasms } \\
\text { Seizures: } \\
\quad \text { - Recurrent } \\
\text { - Resistant to treatment } \\
\text { Developmental delay } \\
\text { Decline in motor skills }\end{array}$ & $\begin{array}{l}\text { Early-onset epileptic encephalopathy } \\
\text { Dystonic movements } \\
\text { Spasticity } \\
\text { Hypotonia } \\
\text { Infantile spasms } \\
\text { Seizures: } \\
\quad \text { - Recurrent } \\
\text { - Pharmaco-resistant } \\
\text { Developmental delay } \\
\text { Decline in motor skills }\end{array}$ \\
\hline Intellectual disability & Severe & Ranging from moderate to severe \\
\hline Optic fundi & Normal & Normal \\
\hline Vision & Difficulty in fixating and following & Impaired fixation, squinting \\
\hline Microcephaly & Yes & Yes $(<-3 S D)$, one case with $-2 S D$ \\
\hline Brain MRI & $\begin{array}{l}\text { Relatively wide sulci } \\
\text { Mild cerebral atrophy }\end{array}$ & $\begin{array}{l}\text { Widening of sylvian fissures (lateral sulcus) } \\
\text { Thin corpus callosum } \\
\text { White matter hyperintensities } \\
\text { Delayed myelination } \\
\text { Mild cerebellar hypoplasia } \\
\text { Mild cortical and central atrophy } \\
\text { Mild cerebral/cerebellar atrophy }\end{array}$ \\
\hline EEG abnormalities & Modified hypsarrhythmia & Ranging from none to hypsarrhythmia \\
\hline
\end{tabular}


Table 2 Annotation of the two UBA5 mutations detected in the sisters

\begin{tabular}{|c|c|c|}
\hline & mutation A (maternal) & mutation B (paternal) \\
\hline Position (GRCh38) & chr3:132,675,903 & chr3:132,671,881 \\
\hline Variant consequence & Missense & Splice region (donor site) leading to a LoF \\
\hline Variant genotype & Heterozygous & Heterozygous \\
\hline cDNA change $e^{a}$ & c.1111G > A (NM_024818.3) & c.684G > A (NM_024818.3) \\
\hline Protein change ${ }^{a}$ & p.Ala371Thr (NP_079094.1) & p.(Ala228=) (NP_079094.1) \\
\hline Transcript length & 404 AA (NM_024818.3) & 404 AA (NM_024818.3) \\
\hline Exon/exons in transcript & 11/12 (NM_024818.3) & 7/12 (NM_024818.3) \\
\hline Allelic frequency Iceland & $0.38 \%$ (249/30,067 Icelanders) & Absent from 30,064 Icelanders \\
\hline Allelic frequency abroad (gnomAD [7]) & $\begin{array}{l}0.58 \%(75 / 12,985 \text { Finnish individuals) } \\
0.25 \%(157 / 62,175 \text { European, } \\
\text { non-Finnish individuals) })^{b}\end{array}$ & $\begin{array}{l}\text { Seen in one individual (European, non-Finnish) } \\
\text { out of } 138,632\end{array}$ \\
\hline $\mathrm{SIFT}^{\mathrm{C}}$ & Deleterious & NA \\
\hline PolyPhen-2 ${ }^{c}$ & Possibly damaging & NA \\
\hline GERP conservation score ${ }^{d}$ & Highly conserved (5.44) & Highly conserved (4.95) \\
\hline Disease in literature & Early Infantile Epileptic Encephalopathy-44 [2-4] & \\
\hline
\end{tabular}

${ }^{a}$ Functional annotation as suggested by the Human Genome Variation Society (HGVS)

${ }^{\mathrm{b}} \mathrm{A}$ lower frequency in other populations

'SIFT and PolyPhen-2 are scores for the predicted effect of amino acid substitution on protein structure and function

${ }^{\mathrm{d}}$ The Genomic Evolutionary Rate Profiling (GERP) framework gives a measure of evolutionary conservation based on the alignment of sequences from 29 mammalian species

of $U B A 5$ with the introduction of a premature stop codon 18 amino acids downstream of the exon/intron boundary of exon 7. Both scenarios would result in nonsensemediated decay of the mature transcript with a corresponding loss-of-function effect. We note that in one of the UBA5 families described previously (by Muona et al.) a $U B A 5$ mutation was detected at the third nucleotide of the acceptor (3') end of exon 2 [4]. That mutation was initially annotated as a missense, p.Arg55His, but was reclassified as a loss-of-function (LoF) mutation when shown to interfere with splicing and result in nonsense-mediated decay [4].

The other mutation detected in $U B A 5$ in the two sisters is a missense mutation, p.Ala371Thr, inherited from their mother. The mutation has a minor allele frequency of $0.38 \%$ in Iceland, $0.58 \%$ in Finnish and $0.25 \%$ in nonFinnish Europeans, with lower frequencies in other populations [7]. The two reports describing the role of biallelic variants in UBA5 in early-onset epileptic encephalopathy classify p.Ala371Thr as a hypomorphic allele that contributes to a severe neurological phenotype only when in trans with a LoF in UBA5 [3, 4]. In our set of 150,656 chip-genotyped Icelanders, into which we have imputed all variants detected in a large set of whole-genome sequenced Icelanders [5], we have identified three individuals who are homozygous for the p.Ala371Thr mutation. We have extensive phenotypic information linked to the set of 150,656 genotyped individuals. All three individuals homozygous for p.Ala371Thr have reached adult age without showing signs of a neurological phenotype, and two of them have three and six children, respectively (Table 3). Our findings confirm the hypomorphic nature of p.Ala371Thr and prove that it is only pathogenic when in combination with a LoF in $U B A 5$.

\section{Discussion and conclusions}

We describe a compound heterozygous genotype in $U B A 5$ in two sisters with early-onset epileptic encephalopathy. The sisters and their parents were initially whole-exome sequenced in 2013. The two compound heterozygous mutations in $U B A 5$ were detected in the whole-exome data but at the time, no disease-causing mutation had been described in this gene and the genotype was therefore not read as a pathogenic one. We decided to repeat the analysis of the family using whole-genome sequencing in 2016. In September 2016 the two reports [3, 4] on biallelic variants

Table 3 Icelandic individuals homozygous for p.Ala371Thr in UBA5

\begin{tabular}{lllll}
\hline Individual & Gender & Year of birth & $\begin{array}{l}\text { Number of } \\
\text { offspring }\end{array}$ & $\begin{array}{l}\text { Main reported } \\
\text { phenotypes }\end{array}$ \\
\hline A & Female & $1934-$ & 3 & $\begin{array}{l}\text { Coronary artery disease } \\
\text { Essential hypertension }\end{array}$ \\
B & Male & $1946-$ & 0 & $\begin{array}{l}\text { Osteoarthritis } \\
\text { C }\end{array}$ \\
Female & $1940-$ & 6 & $\begin{array}{l}\text { Myocardial infarction } \\
\text { Coronary artery disease }\end{array}$ \\
& & & & Ulcerative colitis
\end{tabular}

For the three p.Ala371Thr homozygous individuals detected in the Icelandic reference cohort we show gender, year of birth, number of offspring and main clinical phenotypes. The phenotypes were obtained from the National University Hospital of Iceland's registry. All three individuals are still alive and two of them have children, none of them has a reported neurological condition. Individuals $B$ and $C$ are siblings 
in $U B A 5$ causing early-onset epileptic encephalopathy were published, allowing us to conclude on the pathogenicity of the compound heterozygous mutations in $U B A 5$ in the two affected sisters. The progression of this case underlines the importance of periodically revisiting the most recent literature when performing genetic analysis of clinical cases.

We have identified three adult and unaffected individuals homozygous for one of the mutations detected in the two sisters, p.Ala371Thr, thus confirming that it is hypomorphic and not disease-causing by itself. As we do, the authors of the two reports on biallelic variants in UBA5 describe the combination of p.Ala371Thr and a loss-of-function mutation to cause early-onset epileptic encephalopathy $[3,4]$. The paternally inherited exonic splicing mutation we present is predicted to result in abnormal splicing of $U B A 5$ with a subsequent loss-of-function effect.

$U B A 5$ is the first ufmylation gene identified in epileptic encephalopathies. Although a link between defects in other post-translational modification mechanisms and this disease group has been demonstrated [9], the exact role of impaired ufmylation in epileptic encephalopathy is still unclear. The identification and reporting of new pathogenic $U B A 5$ genotypes adds necessary evidence to the pathogenetic story of $U B A 5$ and defects in the ufmylation process.

\section{Additional files}

Additional file 1: Figure S1. Brain MRI of the older sister. Axial T2 image of the older sister (II-2) showing relatively wide sulci and a mild cerebral atrophy. (DOCX $21 \mathrm{~kb}$ )

Additional file 2: Supplementary methods. A detailed description of the sample acquisition, sample preparations, whole-genome sequencing, alignment, variant calling and annotation. (DOCX 148 kb)

Additional file 3: Figure S2. Variant filtering flowchart. This flowchart depicts how we narrowed nearly 5,000,000 variants from the sequenced genome of each affected sister down to a single shared genotype; compound heterozygous mutations in the gene UBA5. (DOCX $71 \mathrm{~kb}$ )

Additional file 4: Table S1. Sequencing data metrics for the UBA5 missense mutation (p.Ala371Thr) and exonic splice (c.684G > A). From whole-genome sequencing (WGS) and whole-exome sequencing (WES) data: Call ratios of the alternative alleles in sequenced family members (the two sisters and their parents) and the sequencing depths of the reference and alternative alleles of the two mutations. Also shown are results from the Sanger sequencing of the two mutations in all family members, including the two unaffected brothers (II-1 and II-4) who were neither whole-exome nor whole-genome sequenced. (DOCX $87 \mathrm{~kb}$ )

Additional file 5: Figure S3. UBA5 (NM_024818.3). The positions of the exonic splicing mutation (c.684G > A) and the missense mutation (p.Ala371Thr). (DOCX 14 kb)

\section{Abbreviations}

EEG: Electroencephalogram; EIEE44: Early infantile epileptic encephalopathy 44; gnomAD: Genome aggregation database; LoF: Loss of function; MIM: Mendelian inheritance in man; MRI: Magnetic resonance imaging; mRNA: Messenger ribonucleic acid; MTA1: Metastasis associated 1; SNPs: Single nucleotide polymorphisms; UBA5: Ubiquitin like modifier activating enzyme 5

\section{Acknowledgements}

We wish to acknowledge and thank all members of the family described in this report.
Funding

No funding.

\section{Availability of data and materials}

The datasets supporting the conclusions of this article are included within the article (and its additional files).

\section{Authors' contributions}

GAA, BOJ, GS, AO, RPK and SB analyzed the data and wrote the manuscript, SAG, GM and GAT developed the methods for data analysis, JS collected the samples, OTM performed whole-genome sequencing, AJ, ASJ and AS performed Sanger sequencing, DFG and UT designed the study, PS designed the study, analyzed the data and wrote the manuscript, KS designed the study and wrote the manuscript, SEM and RA performed clinical evaluation of the patients. All authors read and approved the final manuscript.

\section{Ethics approval and consent to participate}

The study was approved by the National Bioethics Committee in Iceland. Written, informed consent to participate was obtained for all participants, or their guardians, before blood samples were drawn.

\section{Consent for publication}

All participants, or their guardians, provided written informed consent for publication of medical data, medical imaging and genetic data.

\section{Competing interests}

GAA, BOJ, GS, AO, RPK, SB, SAG, GM, GAT, JS, OTM, AJ, ASJ, AS, DFG, UT, PS and KS are full-time employees of deCODE Genetics/Amgen, Inc. SEM and RA declare no conflict of interest.

\section{Publisher's Note}

Springer Nature remains neutral with regard to jurisdictional claims in published maps and institutional affiliations.

\section{Author details}

'deCODE Genetics/Amgen, Inc., Sturlugata 8, 101 Reykjavik, Iceland. ${ }^{2}$ Department of Pediatrics, Landspitali University Hospital, Reykjavik, Iceland. ${ }^{3}$ School of Engineering and Natural Sciences, University of Iceland, Reykjavik, Iceland. ${ }^{4}$ Faculty of Medicine, University of Iceland, Reykjavik, Iceland. ${ }^{5}$ Department of Genetics and Molecular Medicine, Landspitali University Hospital, Reykjavik, Iceland.

Received: 20 June 2017 Accepted: 11 September 2017

Published online: 02 October 2017

\section{References}

1. McTague A, Howell KB, Cross JH, Kurian MA, Scheffer IE. The genetic landscape of the epileptic encephalopathies of infancy and childhood. Lancet Neurol. 2016;15(3):304-16.

2. Online Mendelian Inheritance in Man, $\mathrm{OMIM}^{\oplus}$. McKusick-Nathans Institute of Genetic Medicine, Johns Hopkins University (Baltimore, MD). 1995-2017. https://www.omim.org/. Accessed May 2017.

3. Colin E, Daniel J, Ziegler A, et al. Biallelic variants in UBA5 reveal that disruption of the UFM1 Cascade can result in early-onset encephalopathy. Am J Hum Genet. 2016;99(3):695-703.

4. Muona M, Ishimura R, Laari A, et al. Biallelic variants in UBA5 link dysfunctional UFM1 ubiquitin-like modifier pathway to severe infantileonset encephalopathy. Am J Hum Genet. 2016;99(3):683-94.

5. Gudbjartsson DF, Helgason H, Gudjonsson SA, et al. Large-scale wholegenome sequencing of the Icelandic population. Nat Genet. 2015;47(5):435-44

6. Sulem $\mathrm{P}$, Helgason $\mathrm{H}$, Oddson $\mathrm{A}$, et al. Identification of a large set of rare complete human knockouts. Nat Genet. 2015;47(5):448-52.

7. Lek M, Karczewski KJ, Minikel EV, et al. Analysis of protein-coding genetic variation in 60,706 humans. Nature. 2016;536(7616):285-91.

8. Duan $R$, Shi $Y, Y u L$, et al. UBA5 mutations cause a new form of autosomal recessive cerebellar ataxia. PLoS One. 2016;11(2):e0149039.

9. Paemka L, Mahajan VB, Ehaideb SN, et al. Seizures are regulated by ubiquitin-specific peptidase 9 X-linked (USP9X), a de-Ubiquitinase. PLoS Genet. 2015;11(3):e1005022. 